Pacific Journal of Mathematics

SOME EXAMPLES OF RIEMANNIAN ALMOST-PRODUC 


\title{
SOME EXAMPLES OF RIEMANNIAN ALMOST-PRODUCT MANIFOLDS
}

\author{
V. MiQUeL
}

\begin{abstract}
Foliations with bundle-like metrics, conformal, minimal and totally geodesic foliations and minimal and geodesic plane fields have been subject to recent study. A. M. Naveira has fitted all these classes into a general scheme and has gotten thirty six classes of riemannian almostproduct manifolds. In this paper we give strict examples of these classes, showing that none of them is vacuous, and that the inclusion relations among them are strict. The basic riemannian manifolds for the construction of these examples are submanifolds of $\mathbf{C}^{n+1}$ and $\mathbf{H}^{n+1}(\mathrm{C}=$ complex field, $\mathbf{H}=$ quaternion field), and we use the canonical complex structures on these vector spaces. Perhaps the most interesting examples are those of minimal foliations which are not totally geodesic foliations.
\end{abstract}

1. Introduction. Some classes of riemannian almost-product manifolds have been extensively studied in the literature. In the beginning, most of the works on this subject dealt with foliations with bundle-like metrics ([6], [17], [15]). Recently, the study of other types of riemannian almost-product manifolds has been initiated: conformal foliations ([14], [23], [11], [12]), minimal and geodesic plane fields ([18], [24]), minimal foliations ([5], [16], [20], [21]), totally geodesic foliations ([2], [9]). Hsu [8], by analogy with the almost-hermitian manifolds, defined some types of riemannian almost-product manifolds in terms of the symmetries of the tensor $\nabla P$, where $\nabla$ is the Levi-Civita connection and $P$ is the almostproduct tensor ( $P^{2}=$ identity). Naveira [13] has fitted all these classes into a general scheme. This has been accomplished by means of a detailed study of a representation of the pertinent group $O(p) \times O(q)$ on a certain vector space. With this method he has gotten thirty six classes of riemannian almost-product manifolds, and has also given a geometric interpretation of some. Carreras [1], along the study of the natural functions on riemannian almost-product manifolds, has shown that this general scheme is, in a reasonable sense, complete. Gil-Medrano [3] and Montesinos [11] have completed the geometric interpretation.

In this note we give concrete examples of some classes. By using them and the behaviour of the classes under a conformal transformation of the metric studied in [3], we obtain examples of the thirty six classes, which shows that the inclusion relations among the classes are strict. 
The plane of the paper is the following:

$\$ 2$ is a résumé of the general scheme of Naveira and an exposition of the notation employed. In $\S 3$ we give a canonical almost-product structure on a real hypersurface in $\mathbf{C}^{n+1}$, characterize the possible different classes by properties of the second fundamental form $h$, and give concrete examples of (TGF, AF), (TGF, $\left.D_{1}\right),\left(\mathrm{F}_{2}, D_{1}\right)$, (TGF, $\left.\mathrm{F}_{1}\right)$-manifolds. In $\S 4$ we give an almost-product structure on a real hypersurface in $\mathbf{H}^{n+1}$ (H = quaternion field), and, following the same scheme as in $\S 3$, we finish the section with concrete examples of (TGF, AF), (TGF, $\left.D_{1}\right),\left(D_{2}, D_{1}\right)$, $\left(D_{1}, D_{1}\right),\left(\Delta, D_{1}\right),\left(\mathrm{TGF}, \mathrm{F}_{1}\right)$-manifolds. In $\$ 5$ we define an almost-product structure on a complex hypersurface in $\mathbf{H}^{n+1}$ and give concrete examples of $\left(\mathrm{F}_{1}, D_{1}\right)$-manifolds. A surprising fact is that, whereas in $\$ \S 3$ and $5 \mathcal{V}$ is always $F_{2}$ or $F_{1}$, in $\S 4 \mathcal{V}$ is $F$ if and only if it is TGF, and in many manifolds $\mathcal{V}$ is not integrable. Occasionally, the proofs involve messy computations. In these situations we have adopted the method of writing them only in the cases occurring in $\$ 3$ which use similar techniques. In $\$ 6$ examples of the thirty six classes are given.

The effective existence of the thirty six classes (showed by the examples) lends plausibility to the study of their general geometrical and topological properties. On the other hand, the examples given in $\S \S 3-5$ can be considered as possible topics on classical differential geometry since they are defined in $\mathbf{C}^{n+1}$ and $\mathbf{H}^{n+1}$.

The examples given in $3.12,4.9$ and $\S 5$ have a special interest. In fact, as far as I am aware, one encounters in the literature characterizations of minimal foliations ([20], [21], [5]) and sufficient conditions for defining a minimal foliation by a closed form ([16]), but nothing is said to distinguish a minimal foliation from a totally geodesic one.

It is also interesting to remark on the variety of examples of $\mathrm{AF}$ and $D_{1}$ distributions which are not integrable, whilst in [18] only examples on $\mathbf{R}^{3}$ appear.

Finally, the examples given in $\$ 5$ are also examples of complex distributions on Kähler manifolds as defined in [22], and 3.12, 4.9 give examples of closed vector fields on riemannian manifolds as studied in [7].

I wish to thank F. Carreras, A. Ferrández, O. Gil-Medrano, D. L. Johnson, A. M. Naveira and I. Vaisman for useful comments, and especially A. Montesinos who revised the manuscript.

2. Generalities. Let $M$ be a riemannian almost-product manifold with metric tensor $\langle$,$\rangle and almost-product tensor P$. Let $\mathfrak{V}$ be the vertical distribution (generated by the vectors $A$ such that $P A=A$ ), and $\mathcal{H}$ the 
horizontal distribution (generated by the vectors $X$ such that $P X=-X$ ). We also denote by $\mathcal{V}$ and $\mathcal{H}$ the corresponding vector subbundles of the tangent bundle TM. $V$ and $H$ will denote the projections onto $\mathcal{V}$ and $\mathcal{H}$ respectively. Then $P=V-H$.

As shown in [3], the thirty six classes of Naveira can be reobtained by considering on each distribution $\mathscr{D}(\mathcal{V}$ or $\mathcal{H})$ one of the following conditions:

(2.1) AF (anti-foliation): $\nabla_{A}(P) B=-\nabla_{B}(P) A$;

(2.2) $D_{1}$ (minimality): $\sum_{a=1}^{p} \nabla_{E_{a}}(P) E_{a}=0$;

(2.3) $D_{2}$ (umbilicity): $\nabla_{A}(P) A=\frac{1}{p}\langle A, A\rangle \sum_{a=1}^{p} \nabla_{E_{a}}(P) E_{a}$;

(2.4) $\Delta:$ no condition;

(for $A, B \in \mathscr{D},\left\{E_{a}\right\}_{a=1}^{P}$ a local orthonormal frame of $\mathscr{D}$ and $p=$ dimension of $D$ ), and the conditions obtained by adding to them the integrability condition $\mathrm{F}: \nabla_{A}(P) B=\nabla_{B}(P) A$ :

(2.5) TGF (totally geodesic foliation): $\nabla_{A} P=0$;

(2.6) $\mathrm{F}_{1}$ (minimal foliation): minimality and $F$;

(2.7) $\mathrm{F}_{2}$ (umbilical foliation): $\nabla_{A}(P) B=\frac{1}{p}\langle A, B\rangle \sum_{a=1}^{p} \nabla_{E_{a}}(P) E_{a}$;

(2.8) $\mathrm{F}$ (foliation).

There are the following implications

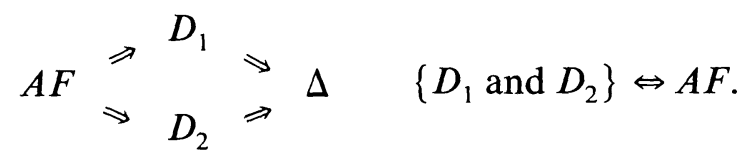

Combining conditions (2.1)-(2.8), eliminating the dual situations, gives the thirty six classes. The inclusion relations among the classes follow from the above implications.

Relations among these classes and the other studied in the literature are given in [3], [11] and [13]. For example, the foliated manifolds with bundle-like metric ([17]) are the (F, AF)-manifolds; the manifolds with conformal foliations ([14]) are the (F, $\left.D_{2}\right)$-manifolds; the manifolds with totally geodesic or minimal plane fields $([18])$ are the $(\mathrm{AF}, \Delta)$-manifolds and the $\left(D_{1}, \Delta\right)$-manifolds respectively.

We shall say that $M$ is $(\mathscr{D}, \mathscr{D})$ strict if ' $\mathfrak{V}$ is $\mathscr{D}, \mathcal{H}$ is $\mathscr{D}$ ' and $M$ is not in any other class included in $\left(\mathscr{D}, \mathscr{D}^{\prime}\right)$.

From now on we denote by $A, B, C, \cdots$ vertical vector fields, by $X$, $Y, Z, \ldots$ horizontal vector fields, and by $S, T, U, \ldots$ arbitrary vector fields on $M .\left\{E_{a}\right\}_{a=1}^{p}$ will denote an orthonormal frame in $\mathscr{V}$ and $\left\{E_{u}\right\}_{u=1}^{q}$ an orthonormal frame in $\mathcal{H}$.

For the real submanifolds in $\mathbf{R}^{m}$ we use the same notations as in [10], except for $A$ which here is denoted $\mathbb{Q}$. 
3. Canonical almost-product structures on real hypersurfaces in $\mathbf{C}^{n+1}$. Let $M$ be an oriented real hypersurface in $\mathbf{C}^{n+1}, N$ the unitary normal vector field on $M$, and $J$ the canonical almost-complex structure on $\mathbf{C}^{n+1}$. These elements define a canonical almost-product structure on $M$, namely that for which $\mathscr{V}=\{J N\}$ and $\mathcal{K}=\{J N\}^{\perp}$ (orthogonal complement of $J N$ in $T M$ ). Observe that $J \mathscr{H}=\mathscr{H}$.

In this section $M$ will denote such an hypersurface in $\mathbf{C}^{n+1}$ with the metric $\langle$,$\rangle induced from that of \mathbf{C}^{n+1}$ and the almost-product tensor $P$ defined above.

First, we observe that the dimension of $\mathscr{V}$ is one, hence $M$ is in the class $\left(\mathrm{F}_{2}, \Delta\right)$. Moreover:

\subsection{Proposition.}

$$
\begin{gathered}
\nabla_{T}(P) X=2 h(T, J X) J N \\
\nabla_{T}(P) J N=-2\{J \& T+h(T, J N) N\} .
\end{gathered}
$$

Proof. $\nabla_{T}(P) X=-\nabla_{T} X-P \nabla_{T} X=-2 V \nabla_{T} X$, and

$$
V \nabla_{T} X=\left\langle\nabla_{T} X, J N\right\rangle J N=\left\langle\nabla_{T}^{\prime} X, J N\right\rangle J N=-\left\langle\nabla_{T}^{\prime}(J X), N\right\rangle J N,
$$

so (3.1) follows. Analogously, $\nabla_{T}(P) J N=2 H \nabla_{T}(J N)$, and a similar computation gives (3.2).

\subsection{Lemma. Every such $M$ is in the class $\left(\mathrm{F}_{2}, D_{1}\right)$.}

Proof. Since it is in $\left(\mathrm{F}_{2}, \Delta\right)$, the class $\left(\mathrm{F}_{2}, D_{1}\right)$ is characterized by (2.2). The condition $J \mathcal{H}=\mathscr{H}$ shows that an orthonormal frame $\left\{E_{u}\right\}$ in $\mathcal{H}$ can be chosen in the form $\left\{E_{1}, \ldots, E_{n}, J E_{1}, \ldots, J E_{n}\right\}$. Then, using (3.1),

$$
\begin{aligned}
\sum_{u=1}^{2 n} \nabla_{E_{u}}(P) E_{u} & =2 \sum_{u=1}^{2 n} h\left(E_{u}, J E_{u}\right) J N \\
& =2 \sum_{u=1}^{n} h\left(E_{u}, J E_{u}\right)-2 \sum_{u=1}^{n} h\left(J E_{u}, E_{u}\right) J N=0
\end{aligned}
$$

Notice that this lemma could be expected, since if $\mathcal{K}$ is integrable, $J \mathcal{H}=\mathcal{K}$ implies that the integral leaves of $\mathcal{K}$ are complex submanifolds of $\mathbf{C}^{n+1}$, hence they are minimal in $\mathbf{C}^{n+1}$. Lemma 3.2 further states they are also minimal in $M$ and the minimality remains when the integrability is dropped.

3.3. Corollary. $\mathcal{H}$ is $F$ iff it is $F_{1}$. 
3.4. Proposition. We have the following equivalences:

(a) $M$ is $\left(\mathrm{F}_{2}, \mathrm{TGF}\right)$ iff $h(X, Y)=0$.

(b) $M$ is (TGF, AF) iff $h(X, J X)=0$ and $h(X, J N)=0$.

(c) $M$ is (TGF, $\left.\mathrm{F}_{1}\right)$ iff $h(X, J Y)=h(J X, Y)$ and $h(X, J N)=0$.

(d) $M$ is $\left(\mathrm{F}_{2}, \mathrm{AF}\right)$ iff $h(X, J X)=0$.

(e) $M$ is $\left(\mathrm{F}_{2}, \mathrm{~F}_{1}\right)$ iff $h(X, J Y)=h(J X, Y)$.

(f) $M$ is (TGF, $\left.D_{1}\right)$ iff $h(X, J N)=0$.

Notice that the condition $h(X, J X)=0$ says $X$ and $J X$ are conjugated directions, $h(X, J N)=0$ says $J N$ is a principal direction and $h(X, J Y)=h(J X, Y)$ is a condition verified by the complex submanifolds of a Kähler manifold.

Proof. (d) and (e) follow immediately from the defining conditions (§2) and (3.1). Now we prove (f). Here, the class (TGF, $D_{1}$ ) is characterized by $\nabla_{J N} P=0$, which, by (3.1), is equivalent to

$h(J N, J X)=0$ for every $X \in \mathcal{H}$ and $J \mathscr{Q} J N+h(J N, J N) N=0$, but

$$
\mathcal{Q} J N=\sum_{u=1}^{2 n} h\left(J N, E_{u}\right) E_{u}+h(J N, J N) J N,
$$

and

$h(J N, J X)=0 \quad$ for every $X \in \mathcal{H} \quad$ iff $h\left(J N, E_{u}\right)=0, \quad u=1, \ldots, 2 n$.

Then $h(J N, J X)=0$ implies $J \& J N+h(J N, J N) N=0$, and (f) is proved. (a), (b), (c) are consequence of (d), (e), (f) and the inclusion relations among the classes.

3.5. Corollary. If $M$ is compact and $b_{1}(M)=0\left(b_{1}(M)=\right.$ first Betti number of $M)$, then $M$ is not (TGF, $\left.F_{1}\right)$.

Proof. Let $\theta$ be the one-form on $M$ given by $\theta(U)=\langle J N, U\rangle$. If $M$ were (TGF, $\left.\mathrm{F}_{1}\right)$, we would have $d \theta(X, Y)=0=d \theta(J N, J N)$ and, by $3.4(\mathrm{c})$,

$$
\begin{aligned}
d \theta(X, J N) & =\nabla_{X}(\theta) J N-\nabla_{J N}(\theta) X=-\left\langle\nabla_{X} J N, J N\right\rangle+\left\langle\nabla_{J N} X, J N\right\rangle \\
& =-h(J N, J X)=0,
\end{aligned}
$$

so $\theta$ would be closed. As $b_{1}(M)=0$ there would exist a real function $f$ on $M$ such that $\theta=d f$. Since $M$ is compact it would be some point $x \in M$ 
where $\theta_{x}=d f(x)=0$, whence $(J N)_{x}=0$. That would yield a contradiction.

The following corollaries give more concrete examples.

3.6. Corollary. Let $M$ be an umbilical real hypersurface in $\mathbf{C}^{n+1}$. Then $M$ is (TGF, AF) strict iff $M$ is an open set of a sphere, and it is locally-product iff $M$ is an open set of a real hyperplane in $\mathbf{C}^{n+1}$.

Proof. The second assertion is obvious. For the first, if $M$ is umbilical, there exists a real number $\lambda$ such that $\mathscr{Q} X=\lambda X$ (which implies $h(X, J X)$ $=0$ ) and $\mathscr{C} J N=\lambda J N$ (which implies $h(J N, X)=0$ ). Then $M$ is (TGF, AF), and it is not locally-product since $h(X, X)=\lambda\|X\|^{2} \neq 0$.

Notice that the canonical almost-product structure on $S^{2 n+1} \subset \mathbf{C}^{n+1}$ coincides with that given by the Hopf fibrations

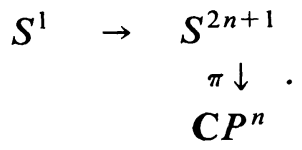

From this viewpoint it is clear that $S^{2 n+1}$ is (TGF, AF). In fact the fibers $S^{1}$ are geodesics of $S^{2 n+1}$, and $S^{2 n+1} \stackrel{\pi}{\rightarrow} \mathrm{C} P^{n}$ is a riemannian submersion; then ([1]) $\nabla_{X}(P) Y=2 \vartheta_{X} Y$, where $\theta$ is the horizontal configuration tensor, which verifies $\theta_{X} X=0([4]$ and [15]).

3.7. Corollary. $M$ is $\left(\mathrm{F}_{2}, \mathrm{TGF}\right)$ iff $\mathcal{H}$ is integrable and its leaves are real $2 n$-planes in $\mathbf{C}^{n+1}$. Then $M$ is an open set of vector bundle $M^{\prime}$ over a curve $\sigma$ in $\mathbf{C}^{n+1}$ such that the fibres are complex hyperplanes in $\mathbf{C}^{n+1}$. Moreover $M^{\prime}$ is contained in the normal vector bundle of $\sigma$ in $\mathbf{C}^{n+1}$.

Proof. If $\mathcal{H}$ is TGF, then the second fundamental form $\alpha$ of an integral leaf of $\mathcal{H}$ viewed as a submanifold in $\mathbf{C}^{n+1}$ is

$$
\begin{aligned}
\alpha(X, Y) & =\left\langle\nabla_{X}^{\prime} Y, N\right\rangle N+\left\langle\nabla_{X}^{\prime} Y, J N\right\rangle J N \\
& =h(X, Y) N-h(X, J Y) J N=0 .
\end{aligned}
$$

Then this leaf is a linear submanifold in $\mathbf{C}^{n+1}$.

3.8. Corollary. A minimal real hypersurface in $\mathbf{C}^{2}$ is $\left(\mathrm{F}_{2}, \mathrm{~F}_{1}\right)$ iff $h(J N, J N)=0$ (i.e. $J N$ is an asymptotic direction). 
From now on we consider two identifications between $\mathbf{C}^{n+1}$ and $\mathbf{R}^{2 n+2}$ : those given by the isomorphisms $j_{1}, j_{2}: \mathbf{C}^{n+1} \rightarrow \mathbf{R}^{2 n+2}$ such that $j_{1}\left(z_{1}, \ldots, z_{n+1}\right)=\left(x_{1}, \ldots, x_{n+1}, y_{1}, \ldots, y_{n+1}\right)$ and $j_{2}\left(z_{1}, \ldots, z_{n+1}\right)=$ $\left(x_{1}, y_{1}, \ldots, x_{n+1}, y_{n+1}\right)$, where $z_{k}=x_{k}+i y_{k}$.

3.9. Proposition. If we consider the identification $j_{1}$ between $\mathbf{C}^{n+1}$ and $\mathbf{R}^{2 n+2}$, we have:

(a) $S^{r} \times \mathbf{R}^{t}(r+t=2 n+1, r \neq 0 \neq t)$ is

(i) (TGF, $\left.D_{1}\right)$ strict for $r \leq n$;

(ii) $\left(\mathrm{F}_{2}, D_{1}\right)$ strict for $n<r \leq 2 n$.

(b) If $M$ is the hyperboloid of one sheet, $\left\{\left(x_{1}, \ldots, x_{2 n+2}\right) \in \mathbf{C}^{n+1} \mid\right.$ $\left.\sum_{l=1}^{2 n+1} x_{i}^{2}-x_{2 n+2}^{2}=1\right\}$, then $M$ is $\left(\mathrm{F}_{2}, D_{1}\right)$ strict.

For the identification $j_{2}$ we have

(c) $S^{r} \times \mathbf{R}^{t}(r, t$ as above) is

(iii) (TGF, AF) strict for $r=2 s-1 \neq 1$ and locally-product iff $r=1$;

(iv) $\left(\mathrm{F}_{2}, D_{1}\right)$ strict for $r=2 s$.

(d) If $M$ is the hyperboloid of one sheet, it is $\left(\mathrm{F}_{2}, D_{1}\right)$ strict.

Proof. (a) At $x=\left(x_{1}, \ldots, x_{r+1}, u_{1}, \ldots, u_{t}\right) \in S^{r} \times \mathbf{R}^{t}, N_{x}=$ $\left(x_{1}, \ldots, x_{r+1}, 0, \ldots, 0\right)$.

(i) In the case $r \leq n, J N_{x}=\left(0, \ldots, 0, x_{1}, \ldots, x_{r+1}, 0, \ldots, 0\right)$ with $x_{1}$ in the $(n+2)$ th spot, and $\mathscr{Q} J N=-\nabla_{J N}^{\prime} N=0$. Then $\mathcal{V}$ is TGF. Moreover, if $X=\left(X_{1}, \ldots, X_{2 n+2}\right) \in \mathcal{H}$, then $\mathbb{Q} X=\left(-X_{1}, \ldots,-X_{r+1}, 0, \ldots, 0\right)$, and $h(X, J Y)=X_{1} Y_{n+2}+\cdots+X_{r+1} Y_{n+r+2}$. At $x=(1,0, \ldots, 0)$, we take $X_{x}$ $=(0,1,0, \ldots, 0,1,0, \ldots, 0)$ with the second 1 in the $(n+3)$ th spot, then $h(X, J X)=1 \neq 0$ and $\mathcal{H}$ is not AF. Similarly, if $X_{x}=(0,1,0, \ldots, 0)$ and $Y_{x}=(0, \ldots, 0,1,0, \ldots, 0)$, with 1 in the $(n+3)$ th spot, then $h(X, J Y)=1$ and $h(Y, J X)=0$, whence $\mathcal{H}$ is not $F_{1}$.

(ii) In the case $r>n, J N=\left(-x_{n+2}, \ldots,-x_{r+1}, 0, \ldots, 0, x_{1}, \ldots, x_{n+1}\right)$ and $\mathscr{Q} J N=\left(-x_{n+2}, \ldots,-x_{r+1}, 0, \ldots, 0, x_{1}, \ldots, x_{r-n}, 0, \ldots, 0\right)$, which shows that $Q J N$ is not proportional to $J N$ in many points $x$ of $S^{r} \times \mathbf{R}^{t}$, since $t \neq 0$ implies $r-n \neq n+1$. Then $\mathscr{V}$ is not TGF. Similar computations as in (i) show that $\mathcal{H}$ is not AF nor $F_{1}$.

(b) If

$$
f\left(x_{1}, \ldots, x_{2 n+2}\right)=\sum_{i=1}^{2 n+1} x_{i}^{2}-x_{2 n+2}^{2}-1
$$

then

$$
N=\frac{\operatorname{grad} f}{2 \sqrt{1+2 x_{2 n+2}^{2}}},
$$


and the proof of (b) follows from similar, but longer, computations than in (a).

(c)(iii) For $r=2 s-1, J N=\left(-x_{2}, x_{1}, \ldots,-x_{2 s}, x_{2 s-1}, 0, \ldots, 0\right), h$ is the same for $S^{r} \times \mathbf{R}^{t}$ in $\mathbf{R}^{2 n+2}$ as for $S^{r}$ in $\mathbf{R}^{2 s}$. Then, if $r \neq 1, S^{r} \times \mathbf{R}^{t}$ is (TGF, AF) strict from 3.6, and, for $r=1, J N$ is the tangent vector to $S^{1}$, whence the result.

(iv) For $r=2 s, J N=\left(-x_{2}, x_{1}, \ldots,-x_{2 s}, x_{2 s-1}, 0, x_{2 s+1}, 0, \ldots, 0\right)$ and Q $J N=\left(x_{2},-x_{1}, \ldots, x_{2 s},-x_{2 s-1}, 0, \ldots, 0\right)$, which is not in the direction of $J N$ in the points wiht $x_{2 s+1} \neq 0$. Then $\mathcal{V}$ is not TGF. Moreover, analogously to (a)(i), $\mathcal{H}$ is not $F_{1}$ nor AF.

(d) Similar computations.

The following is an example where $M$ is compact and the distributions verify the conditions as weakly as possible (cf. 3.2).

3.10. Let $M$ be $S^{2} \times S^{1}$ immersed in $\mathbf{R}^{4}$ by the parametrization $x(\theta, \phi, v)$

$$
=((\cos \theta \cos \phi+a) \cos v,(\cos \theta \cos \phi+a) \sin v, \cos \theta \sin \phi, \sin \theta),
$$

where $a>1$. The entries of the matrix of $h,\left(h_{i j}\right)$, and $\langle\rangle,,\left(g_{i j}\right)$, obey the following relations:

(i) $h_{\theta \theta}=-g_{\theta \theta}, h_{\phi \phi}=-g_{\phi \phi}, h_{v v}=-\left(g_{v v}-a(\cos \theta \cos \phi+a)\right)$,

(ii) $h_{\theta \phi}=h_{\theta v}=h_{\phi v}=g_{\theta \phi}=g_{\theta v}=g_{\phi v}=0$.

A long computation using (i) and (ii) shows that $M$ is $\left(\mathrm{F}_{2}, D_{1}\right)$ strict with the identification $j_{1}$.

3.11. REMARK. All computations are valid if we have, instead of $\mathbf{C}^{n+1}$, a Kähler manifold $M^{\prime}$ of complex dimension $n+1$ and $M$ is a real hypersurface in $M^{\prime}$ with a global unitary normal vector field $N$. In this situation the Corollary 3.6 holds for umbilical real hypersurfaces which are not necessarily spheres.

3.12. Examples of (TGF, $\mathrm{F}_{1}$ )-manifolds can be obtained as follows. Let $M^{\prime}$ be a complex hypersurface of $\mathbf{C}^{n+1}$ with trivial normal vector bundle (for example, let $\boldsymbol{M}^{\prime}$ be the zeros of a holomorphic function $f$ : $\mathbf{C}^{n+1} \rightarrow \mathbf{C}$ of complex rank one on every $\left.\left(z_{1}, \ldots, z_{n+1}\right) \in f^{-1}(0)\right)$ and compact closure. Then we can choose an orthonormal frame $\{N, J N\}$ in the normal vector bundle over $M^{\prime}$. Let $M^{\prime \prime}$ be a tubular neighborhood of $M^{\prime}$ in $\mathbf{C}^{n+1}$ and $\kappa$ a strictly positive real function on $M^{\prime}$ such that $x \pm \kappa(x) J N_{x} \in M^{\prime \prime}$ for every $x \in M^{\prime}$. We define $M=\left\{x+\lambda J N \mid x \in M^{\prime}\right.$ and $|\lambda|<\kappa(x)\}$. Then $M$ is a real hypersurface in $\mathbf{C}^{n+1}$ such that, with the 
canonical almost-product structure, the distribution $\mathcal{H}$ is integrable and the leaves of this foliation are the submanifolds obtained from $M^{\prime}$ by the flow of $J N$, and the vertical leaves are segments $x+\lambda J N,-\kappa(x)<\lambda<$ $\kappa(x)$. Then $\mathcal{V}$ is TGF and $\mathcal{H}$ is $\mathrm{F}_{1}$. Moreover $M$ is locally product iff $M^{\prime}$ is a linear submanifold of $\mathbf{C}^{n+1}$ (see proof of 3.7).

4. Almost product structures on real hypersurfaces in $\mathbf{H}^{n+1}$. Let $M$ be an oriented real hypersurface in $\mathbf{H}^{n+1}, N$ the unitary normal vector field on $M$, and $J_{1}, J_{2}, J_{3}$ the canonical almost-complex structures on $\mathbf{H}^{n+1}$ such that $J_{1} J_{2}=J_{3}$. These elements define an almost-product structure on $M$, namely that for which the vertical distrubution $\mathcal{V}$ is generated by the vector fields $J_{1} N, J_{2} N, J_{3} N$ and $\mathcal{H}=\mathcal{V}^{\perp}$. Observe that $J_{l} \mathcal{H}=\mathcal{H}, i=1,2,3$.

In this section we always consider this almost-product structure on all real hypersurfaces $M$ in $\mathbf{H}^{n+1}$ endowed with the metric induced by the canonical riemannian metric on $\mathbf{H}^{n+1}$.

Proposition. We have the following formulas:

$$
\begin{gathered}
\nabla_{T}(P) Y=2 \sum_{i=1}^{3} h\left(T, J_{i} N\right) J_{i} N \\
\nabla_{T}(P) J_{i} N=-2\left\{J_{l} Q T+h\left(T, J_{i} N\right) N+h\left(T, J_{k} N\right) J_{J} N\right. \\
\left.-h\left(T, J_{J} N\right) J_{k} N\right\},
\end{gathered}
$$

where $\{i, j, k\}$ is a cyclic permutation of $\{1,2,3\}$.

Proof. The formulas are obtained by similar, but longer, computations than in 3.1 .

4.2. Lemma. Every such $M$ is in the class $\left(\Delta, D_{1}\right)$.

Proof. On $M$ we can take a local orthonormal frame in the form $\left\{E_{r}, J_{1} E_{r}, J_{2} E_{r}, J_{3} E_{r}, J_{1} N, J_{2} N, J_{3} N\right\}_{r=1}^{n}$. If we denote $E_{2 r}=J_{1} E_{r}, E_{3 r}=$ $J_{2} E_{r}, E_{4 r}=J_{3} E_{r}$, then $\left\{E_{u}\right\}_{u=1}^{4 n}$ is a local frame in $\mathcal{H}$ and, by (4.1),

$$
\sum_{u=1}^{4 n} \nabla_{E_{u}}(P) E_{u}=2 \sum_{u=1}^{4 n} \sum_{l=1}^{3} h\left(E_{u}, J_{l} E_{u}\right) J_{l} N=0
$$

as can be verified by explicitly writing the summands.

As in 3.2 , this lemma could be expected, and $\mathcal{H}$ is $\mathrm{F}$ iff it is $\mathrm{F}_{1}$. 
4.3. Proposition. On these riemannian almost-product manifolds the conditions $\mathrm{AF}$ or $\mathrm{F}$ on $\mathcal{V}$ are equivalent to the condition TGF.

Proof. $\mathscr{V}$ is AF iff $\nabla_{J_{t} N}(P) J_{i} N=0$. Then, from (4.2), $\left\langle J_{i} \Theta J_{i} N, X\right\rangle=0$, whence $h\left(J_{i} N, X\right)=0$ for every $X \in \mathcal{H}$ because $J_{i} \mathcal{H}=\mathcal{H}$.

$\mathcal{V}$ is $F$ iff $\nabla_{J_{t} N}(P) J_{j} N=\nabla_{J_{1} N}(P) J_{i} N$. Then, from (4.2), $h\left(J_{i} N, J_{j} X\right)$ $=h\left(J_{j} N, J_{i} X\right)$. Therefore, if $\{i, j, k\}$ is a cyclic permutation of $\{1,2,3\}$ and $X=J_{k} Y$, we have $h\left(J_{i} N, J_{i} Y\right)=-h\left(J_{j} N, J_{j} Y\right)$ for every $i, j$, whence $h\left(J_{j} N, J_{j} Y\right)=-h\left(J_{k} N, J_{k} Y\right)=h\left(\mathrm{iJ}_{i} N, J_{i} Y\right)$. Then $h\left(J_{i} N, Z\right)=0$ for every $Z \in \mathcal{H}$.

Finally, $\mathscr{V}$ is TGF iff $\nabla_{J_{i} N} P=0$. Then, from (4.1), $h\left(J_{i} N, J_{i} Y\right)=0$, and, as above, $h\left(J_{i} N, X\right)=0$ for every $X \in \mathcal{H}$. Conversely, if $h\left(J_{i} N, J_{i} Y\right)$ $=0$, then $\nabla_{J_{i} N}(P) Y=0$ and, by the symmetry properties of $\nabla P$, $\nabla_{J_{\imath} N}(P) J_{j} N=0$, so $\mathscr{V}$ is TGF.

4.4. Corollary. Among these riemannian almost-product manifolds we have the following equalities of classes:

(a) $\left(\mathrm{AF}, D_{1}\right)=\left(\mathrm{F}, D_{1}\right)=\left(\mathrm{TGF}, D_{1}\right)$.

(b) $(\mathrm{AF}, \mathrm{AF})=(\mathrm{F}, \mathrm{AF})=(\mathrm{TGF}, A F)$.

(c) $\left(\mathrm{AF}, \mathrm{F}_{1}\right)=\left(\mathrm{F}, \mathrm{F}_{1}\right)=\left(\mathrm{TGF}, F_{1}\right)$.

(d) $(\mathrm{AF}, \mathrm{TGF})=(\mathrm{F}, \mathrm{TGF})=$ locally-product .

Using the equations defining the classes, (4.2) and 4.4 we can get the characterization of all the possible classes of these riemannian almostproduct manifolds in terms of $h$. This is the content of the following proposition:

4.5. Proposition. We have the following equivalence:

(a) $M$ is $(\Delta, \mathrm{AF})$ iff $h\left(X, J_{i} X\right)=0$.

(b) $M$ is $\left(\Delta, \mathrm{F}_{1}\right)$ iff $h\left(X, J_{i} Y\right)=h\left(J_{i} X, Y\right)$.

(c) $M$ is $\left(D_{1}, D_{1}\right)$ iff $\sum_{i=1}^{3} h\left(J_{i} N, J_{i} X\right)=0$.

(d) $M$ is $\left(D_{2}, D_{1}\right)$ iff $h\left(J_{i} N, J_{i} X\right)=h\left(J_{j} N, J_{j} X\right)$.

(e) $M$ is $(\Delta$, TGF) iff $h(X, Y)=0$.

(f) $M$ is $\left(D_{1}, \mathrm{AF}\right)$ iff $h\left(X, J_{i} X\right)=0$ and $\sum_{i=1}^{3} h\left(J_{i} N, J_{i} X\right)=0$.

(g) $M$ is $\left(D_{2}, \mathrm{AF}\right)$ iff $h\left(X, J_{i} X\right)=0$ and $h\left(J_{i} N, J_{i} X\right)=h\left(J_{j} N, J_{j} X\right)$.

(h) $M$ is $\left(D_{1}, \mathrm{~F}_{1}\right)$ iff $h\left(X, J_{i} Y\right)=h\left(J_{i} X, Y\right)$ and $\sum_{i=1}^{3} h\left(J_{i} N, J_{i} X\right)=0$.

(i) $M$ is $\left(D_{2}, \mathrm{~F}_{1}\right)$ iff $h\left(J_{i} N, J_{i} X\right)=h\left(J_{j} N, J_{j} X\right)$ and $h\left(X, J_{i} Y\right)=$ $h\left(J_{i} X, Y\right)$.

(j) $M$ is $\left(D_{1}\right.$, TGF) iff $h(X, Y)=0$ and $\sum_{i=1}^{3} h\left(J_{i} N, J_{i} X\right)=0$.

(k) $M$ is $\left(D_{2}, \mathrm{TGF}\right)$ iff $h(X, Y)=0$ and $h\left(J_{i} N, J_{i} X\right)=h\left(J_{j} N, J_{j} X\right)$.

(l) $M$ is (TGF, $\left.D_{1}\right)$ iff $h\left(J_{i} N, X\right)=0$. 
(m) $M$ is (TGF, AF) iff $h\left(J_{i} N, X\right)=0$ and $h\left(X, J_{i} X\right)=0$.

(n) $M$ is (TGF, $\left.\mathrm{F}_{1}\right)$ iff $h\left(J_{l} N, X\right)=0$ and $h\left(X, J_{l} Y\right)=h\left(J_{l} X, Y\right)$.

Similarly to 3.6 and 3.7 we get

4.6. Corollary. Let $M$ be an umbilical real hypersurface in $\mathbf{H}^{n+1}$. Then it is (TGF, AF) strict iff $M$ is an open set of a sphere, and it is locally-product iff it is an open set of a real hyperplane of $\mathbf{H}^{n+1}$.

As in 3.6 the almost-product structure given here on $S^{4 n+3}$ is also given by the Hopf fibration

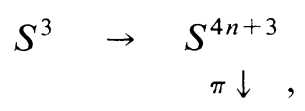

\section{$\mathbf{H} P^{n}$}

and consideration of $S^{4 n+3} \stackrel{\pi}{\rightarrow} \mathbf{H} P^{n}$ as a riemannian submersion also shows that $S^{4 n+3}$ is (TGF, AF).

4.7. Corollary. $M$ is ( $\Delta$, TGF) iff the leaves of the horizontal foliation are complex 2 -planes in $\mathbf{H}^{n+1}$.

We consider the identifications between $\mathbf{H}^{n+1}$ and $\mathbf{R}^{4 n+4}$ given by the isomorphisms $k_{1}, k_{2}: \mathbf{H}^{n+1} \rightarrow \mathbf{R}^{4 n+4}$ such that

$$
\begin{aligned}
& k_{1}\left(q_{1}, \ldots, q_{n+1}\right)=\left(x_{1}, \ldots, x_{n+1}, y_{1}, \ldots, y_{n+1}, u_{1}, \ldots, u_{n+1}, v_{1}, \ldots, v_{n+1}\right), \\
& k_{2}\left(q_{1}, \ldots, q_{n+1}\right)=\left(x_{1}, y_{1}, u_{1}, v_{1}, \ldots, x_{n+1}, y_{n+1}, u_{n+1}, v_{n+1}\right),
\end{aligned}
$$

where $q_{l}=\left(x_{l}, y_{l}, u_{i}, v_{i}\right)$.

After a long computation following the technique of 3.9, we obtain the following concrete examples:

4.8. Proposition. (a) Under the identification $k_{1}, S^{r} \times \mathbf{R}^{t}(r+t=$ $4 n+3, r \neq 0 \neq t)$ is

(i) (TGF, $D_{1}$ ) strict for $r \leq n$ or $r=2 n+1$,

(ii) $\left(\Delta, D_{1}\right)$ strict for $r>n$ and $r \neq 2 n+1$ and $r \neq 3 n+2$,

(iii) $\left(D_{1}, D_{1}\right)$ strict for $r=3 n+2$.

(b) Under the identification $k_{2}, S^{r} \times \mathbf{R}^{t}(r, t$ as above) is

(iv) locally-product for $r=1,2,3$,

(v) $\left(D_{2}, D_{1}\right)$ strict for $r=4 s(s=1,2, \ldots)$,

(vi) $\left(\Delta, D_{1}\right)$ strict for $r=4 s+1,4 s+2$,

(vii) (TGF, AF) strict for $r=4 s+3$. 
4.9. Examples of $\left(\mathrm{TGF}, \mathrm{F}_{1}\right)$ can be given by a similar construction to that of 3.12 , but with $M^{\prime}$ a complex submanifold in $\mathbf{H}^{n+1}$ of complex dimension $2 n$ and with trivial normal vector bundle.

5. Canonical almost product structures on complex hypersurfaces in $\mathbf{H}^{n+1}$. $\quad \mathbf{H}^{n+1}$ can be identified with $\mathbf{C}^{2 n+2}$. Then it is meaningful to consider a complex hypersurface in $\mathbf{H}^{n+1}$. Let $M$ be a such hypersurface with trivial normal vector bundle. We consider as almost-complex structure on $\mathbf{C}^{2 n+2}=\mathbf{H}^{n+1}$ the one given by $J_{1}$. Let $\left\{N, J_{1} N\right\}$ be a frame in the normal vector bundle of $M$. Then we can consider on $M$ the vertical distribution $\mathcal{V}$ generated by $\left\{J_{2} N, J_{3} N\right\}$, and $\mathcal{H}=\mathcal{V}^{\perp}$. This defines an almost-product structure $P$ on $M$. In this section $M$ will represent such a riemannian almost-product manifold. For the components of the real second fundamental form $\alpha$ of $M$, we use the notation:

$$
\alpha(S, T)=h^{0}(S, T) N+h^{1}(S, T) J_{1} N .
$$

As in $\S \S 3$ and 4 we have the following

\subsection{Proposition.}

$$
\begin{gathered}
\nabla_{T}(P) X=2\left\{h^{0}\left(T, J_{2} X\right) J_{2} N+h^{0}\left(T, J_{3} X\right) J_{3} N\right\} . \\
\left\langle\nabla_{T}(P) J_{i} N, X\right\rangle=2 h^{0}\left(T, J_{i} X\right), \quad i=2,3 .
\end{gathered}
$$

\subsection{LemMa. Every such $M$ is in the class $\left(\mathrm{F}_{1}, D_{1}\right)$.}

Proof. We choose a local frame $\left\{E_{r}, J_{1} E_{r}, J_{2} E_{r}, J_{3} E_{r}, J_{2} N, J_{3} N\right\}$ on $T M$. Then $\sum_{u=1}^{4 n} \nabla_{E_{u}}(P) E_{u}=0$ as in 4.2. Moreover, since $M$ is a complex submanifold of $\mathbf{C}^{2 n+2}, \alpha(S, T)=-\alpha\left(J_{1} S, J_{1} T\right)$; then

$$
\begin{aligned}
\left\langle\nabla_{J_{2} N}(P) J_{3} N, X\right\rangle & =2 h^{0}\left(J_{2} N, J_{3} X\right)=-2 h^{0}\left(J_{1} J_{2} N, J_{1} J_{3} X\right) \\
& =2 h^{0}\left(J_{3} N, J_{2} X\right)=\left\langle\nabla_{J_{3} N}(P) J_{2} N, X\right\rangle,
\end{aligned}
$$

and

$$
\begin{aligned}
\left\langle\nabla_{J_{2} N}(P) J_{2} N, X\right\rangle & =2 h^{0}\left(J_{2} N, J_{2} X\right) \\
& =-2 h^{0}\left(J_{3} N, J_{3} X\right)=-\left\langle\nabla_{J_{3} N}(P) J_{3} N, X\right\rangle .
\end{aligned}
$$

As in 3.2 and 4.2 , we could expect that $M$ was $\left(D_{1}, D_{1}\right)$. The agreeable fact is that $\mathfrak{V}$ is also $F$. 
As in 3.4 and 4.5 , the different classes of almost-product structures can be characterized by symmetries of $h^{0}$, but here $h^{0}$ is not the whole second fundamental form of $M$.

We identify $\mathbf{H}^{n+1}$ with $\mathbf{C}^{2 n+2}$ by the isomorphism $k: \mathbf{H}^{n+1} \rightarrow \mathbf{C}^{2 n+2}$ such that $k\left(q_{1}, \ldots, q_{n+1}\right)=\left(z_{1}, z_{2}, \ldots, z_{2 n+1}, z_{2 n+2}\right)$ (where $q_{i}=$ $\left(z_{2 i-1}, z_{2 i}\right)$ ), and with $\mathbf{R}^{4 n+4}$ by $j_{2} \circ k$, where $j_{2}$ is the isomorphism given in $\S 3$. Then:

5.3. Proposition. If $r$ is a real number, $M=\left\{\left(z_{1}, \ldots, z_{2 n+2}\right) \in\right.$ $\left.\mathbf{H}^{n+1} / \sum_{i=1}^{2 n+2} z_{i}^{2}=r\right\}$ is $\left(\mathrm{F}_{1}, D_{1}\right)$-strict.

Proof. First, we observe that if $f\left(z_{1}, \ldots, z_{2 n+2}\right)$ is a holomorphic function and $\nabla f=\left(\partial f / \partial z_{1}, \ldots, \partial f / \partial z_{2 n+2}\right)$, then $\left\{\overline{\nabla f}, J_{1} \overline{\nabla f}\right\}$ is a frame in the normal vector bundle of $M$. With this remark, the proof is a computation similar to those in 3.9 .

6. Examples of the thirty six classes. In $\S \S 3-5$ we gave examples of nine classes of riemannian almost-product manifolds. Here, our point is to give strict examples for the thirty six classes. This proves that the inclusions among the classes are strict. In order to get all of them, it is enough to build a few because the rest can be obtained by using the following results:

6.1. Proposition ([3]). Let $(M ;\langle\rangle$,$) be a riemannian manifold, \mathscr{D}$ a distribution on $M, g$ a real function on $M$ and $\langle,\rangle^{\circ}=e^{2 g}\langle$,$\rangle . Then:$

(a) If $\mathscr{D}$ is $\mathrm{AF}$ for $\left\langle\right.$, , , then it is $D_{2}$ for $\langle,\rangle^{\circ}$, and it is $\mathrm{AF}$ for $\langle,\rangle^{\circ}$ iff $X g=0$ for every $X \in \mathscr{Q}^{\perp}$.

(b) If $\mathscr{D}$ is $D_{1}$ for $\langle$,$\rangle , then it is \Delta$ for $\langle,\rangle^{\circ}$, it is $D_{1}$ for $\langle,\rangle^{\circ}$ iff $X g=0$ for every $X \in \mathscr{D}^{\perp}$ and it is $D_{2}$ for $\langle,\rangle^{\circ}$ iff it is $\operatorname{AF}$ for $\langle$,$\rangle .$

(d) If $\mathscr{Q}$ is $D_{2}$ for $\langle$, $\rangle$, then it is also $D_{2}$ for $\langle,\rangle^{\circ}$.

6.2. Proposition. Let $M$ be a $(\mathrm{F}, \Delta)$-manifold. Then there is a real function $f$ on a open set of $M$ such that $A f=0$ for every $A \in \mathcal{V}$ and $X f \neq 0$ for some $X \in \mathcal{H}$.

6.3. Proposition. Let $M, M^{\prime}$ be two riemannian manifolds, of $a$ distribution on $M$ and $\mathscr{D}^{\prime}$ another one on $M^{\prime}$. On the riemannian product $M \times M^{\prime}$ we consider the distribution $\mathscr{D} \oplus \mathscr{D}^{\prime}$. If $\mathscr{D}$ and $\mathscr{D}^{\prime}$ are both $\mathrm{AF}, D_{1}$ or $\mathrm{F}$, then $\mathscr{D} \oplus \mathscr{D}^{\prime}$ is $\mathrm{AF}, D_{1}$ or $\mathrm{F}$, respectively. 
Proof. It is a consequence of the formula

$$
\nabla_{A+A^{\prime}}^{\prime \prime}\left(P+P^{\prime}\right)\left(B+B^{\prime}\right)=\nabla_{A}(P) B+\nabla_{A^{\prime}}^{\prime}\left(P^{\prime}\right) B^{\prime},
$$

where $P, P^{\prime}, P+P^{\prime}$ are the almost-product tensors corresponding to the structures $\left(\mathscr{D}, \mathscr{D}^{\perp}\right),\left(\mathscr{D}^{\prime}, \mathscr{Q}^{\prime \perp}\right),\left(\mathscr{D} \oplus \mathscr{D}^{\prime},\left(\mathscr{D} \oplus \mathscr{D}^{\prime}\right)^{\perp}\right)$ on the manifolds $M$, $M^{\prime}, M \times M^{\prime}$, respectively, and $\nabla, \nabla^{\prime}, \nabla^{\prime \prime}$ are their corresponding LeviCivita connections.

6.4. Proposition. Let $(M, P)$ be a product manifold and $\left(M^{\prime}, P^{\prime}\right)$ a riemannian almost-product structure. Then, in the induced almost-product structure $\left(M \times M^{\prime}, P+P^{\prime}\right)$ there exists a real function $g$ such that $X g=0$ for every $X \in \mathcal{H} \oplus \mathcal{H}^{\prime}$ and $A g \neq 0$ for some $A \in \mathcal{V} \oplus \mathcal{V}^{\prime}$, and another $f$ such that $A f=0$ for every $A$ and $X f \neq 0$ for some $X$.

Proof. Let $M=\mathscr{B} \times \mathscr{M}$ and $g^{\prime}: \mathscr{B} \rightarrow \mathbf{R}$ not constant, take $g(x, y, z)$ $=g^{\prime}(x)$ for $(x, y, z) \in \Re \times \Re \times M^{\prime}$. With respect to $f$ the proof is similar.

Now we give the examples. First, we note that, by 6.1 and 6.2 or 6.4 , it is enough to have examples for which the distributions are TGF, $F_{1}, A F$ or $D_{1}$. We give a list of these examples:

(TGF, TGF) product manifolds.

(TFG, $F_{1}$ ) examples given in 3.12 and 4.9 .

(TGF, AF) examples given in 3.6 and 4.6.

(TGF, $D_{1}$ ) examples given in 3.9(i) and 4.8(i).

$\left(\mathrm{F}_{1}, \mathrm{~F}_{1}\right) \quad\left(M \times M^{\prime}, P+P^{\prime}\right)$, where $(M, P)$ is $\left(\mathrm{F}_{1}, \mathrm{TGF}\right)$ and $\left(M^{\prime}, P^{\prime}\right)$ is (TGF, $\left.\mathrm{F}_{1}\right)$.

$\left(\mathrm{F}_{1}, \mathrm{AF}\right) \quad\left(M \times M^{\prime}, P+P^{\prime}\right)$, where $(M, P)$ is $\left(\mathrm{F}_{1}, \mathrm{TGF}\right)$ and $\left(M^{\prime}, P^{\prime}\right)$ is (TGF, AF).

$\left(\mathrm{F}_{1}, D_{1}\right) \quad\left(M \times M^{\prime}, P+P^{\prime}\right)$, where $(M, P)$ is $\left(\mathrm{F}_{1}, \mathrm{TGF}\right)$ and $\left(M^{\prime}, P^{\prime}\right)$ is (TGF, $\left.D_{1}\right)$; and examples given in 5.3.

(AF, AF) $\quad\left(M \times M^{\prime}, P+P^{\prime}\right)$, where $(M, P)$ is (AF, TGF) and $\left(M^{\prime}, P^{\prime}\right)$ is (TGF, AF).

$\left(\mathrm{AF}, D_{1}\right) \quad\left(M \times M^{\prime}, P+P^{\prime}\right)$, where $(M, P)$ is (AF, TGF) and $\left(M^{\prime}, P^{\prime}\right)$ is (TGF, $\left.D_{1}\right)$.

$\left(D_{1}, D_{1}\right) \quad\left(M \times M^{\prime}, P+P^{\prime}\right)$, where $(M, P)$ is $\left(D_{1}\right.$, TGF $)$ and $\left(M^{\prime}, P^{\prime}\right)$ is (TGF, $\left.D_{1}\right)$; and examples given in 4.8(iii). 
The examples in the list given as products of almost-product manifolds are correct by 6.3 .

\section{REFERENCES}

[1] F. Carreras, Linear invariants of riemannian almost-product manifolds, Math. Proc. Cambridge Phil. Soc., 91 (1982), 99-106.

[2] P. Dombrowski, Jacobi fields, totally geodesic foliations and geodesic differential forms, Resultate der Mathematic, 1 (1978), 156-194.

[3] O. Gil-Medrano, On the geometric properties of some classes of almost product structures, Rendiconti Circ. Mat. di Palermo, (1984).

[4] A. Gray, Pseudo-Riemannian almost product manifolds and submersions, J. Math. Mech., 16 (1967), 715-737.

[5] A. Haefliger, Some remarks on foliations with minimal leaves, J. Differential Geometry, 15 (1980), 269-284.

[6] R. Hermann, On the differential geometry of foliations, Annals of Math., 72 (1960), 445-457.

[7] N. Hicks, Closed vector fields, Pacific J. Math., 15 (1965), 141-151.

[8] C. J. Hsu, Remarks on certain almost-product spaces, Pacific J. Math., 14 (1964), 163-176.

[9] D. L. Johnson and L. B. Whitt, Totally geodesic foliations, J. Differential Geometry, 15 (1980), 225-235.

[10] S. Kobayashi and K. Nomizu, Foundations of differential geometry, II, Interscience Publishers (1969).

[11] A. Montesinos, On certain classes of almost-product structures, Michigan Math. J., 30 (1983), 31-36.

[12] Conformal curvature for the normal bundle of a conformal foliation, Ann. Inst. Fourier, 32 , 3 (1982), 261-274.

[13] A. M. Naveira, A classification of riemannian almost-product manifolds, Rendiconti di Mat. di Roma (to appear).

[14] S. Nishikawa and H. Sato, On characteristic classes of riemannian, conformal and projective foliations, J. Math. Soc. Japan, 28, 2 (1976), 223-241.

[15] B. O'Neill, The fundamental equations of a submersion, Michigan Math. J., 13 (1966), 459-469.

[16] G. I. Oshikiri, A remark on minimal foliations, Tohoku Math. J. 33 (1981), 133-137.

[17] B. L. Reinhart, Foliated manifolds with bundle-like metrics, Ann. of Math., 69 (1959), 119-131.

[18] - The second fundamental form of a plane field, J. Differential Geometry, 12 (1977), 619-627.

[19] Foliations and second fundamental forms, Proc. of the IV International Colloquium on Diff. Geom., Santiago de Compostela, (1978), 246-253.

[20] H. Rummler, Quelques notions simplex en géométrie riemannienne et leurs applications aux feuilletages compacts, Comment. Math. Helvetici, 54 (1979), 224-239.

[21] D. Sullivan, $A$ homological characterization of foliations consisting of minimal surfaces, Comment. Math. Helvetici, 54 (1979), 218-223.

[22] I. Vaisman, On the analytic distributions and foliations of a Kahler manifolds, Proc. Amer. Math. Soc., 58 (1976), 221-228. 
[23] __ Conformal foliations, Kodai Math. J., 2 (1979), 26-37.

[24] E. Vidal and E. Vidal-Costa, Special connections and almost foliated metrics. J. Differential Geometry, 8 (1973), 297-304.

Received February 16, 1982.

UNIVERSIDAD DE VALENCIA

DePartamento de Geometría y TOPOlogía

Facultad de Ciencias Matemáticas

Burjasot (Valencia) Spain 


\section{PACIFIC JOURNAL OF MATHEMATICS}

EDITORS

Donald BabBITT (Managing Editor)

University of California

Los Angeles, CA 90024

Hugo Rossi

University of Utah

Salt Lake City, UT 84112

C. C. Moore and Arthur Ogus

University of California

Berkeley, CA 94720
J. DugunduI

Department of Mathematics

University of Southern California

Los Angeles, CA 90089-1113

R. FINN and H. SAMELSON

Stanford University

Stanford, CA 94305

\section{ASSOCIATE EDITORS}
R. ARENS
E. F. BECKENBACH
B. H. NeumanN
F. WolF
K. YosHIDA (1906-1982)

\section{SUPPORTING INSTITUTIONS}

UNIVERSITY OF ARIZONA

UNIVERSITY OF BRITISH COLUMBIA

CALIFORNIA INSTITUTE OF TECHNOLOGY

UNIVERSITY OF CALIFORNIA

MONTANA STATE UNIVERSITY

UNIVERSITY OF NEVADA. RENO

NEW MEXICO STATE UNIVERSITY

OREGON STATE UNIVERSITY
UNIVERSITY OF OREGON

UNIVERSITY OF SOUTHERN CALIFORNIA

STANFORD UNIVERSITY

UNIVERSITY OF HAWAII

UNIVERSITY OF TOKYO

UNIVERSITY OF UTAH

WASHINGTON STATE UNIVERSITY

UNIVERSITY OF WASHINGTON 


\section{Pacific Journal of Mathematics}

\section{Vol. 111, No. $1 \quad$ November, 1984}

Harald Brandenburg and Adam Stefan Mysior, For every Hausdorff

space $Y$ there exists a nontrivial Moore space on which all continuous

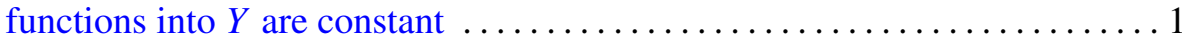

Henry Dappa, A Marcinkiewicz criterion for $L^{p}$-multipliers $\ldots \ldots \ldots \ldots \ldots 9$

P. H. Doyle, III and John Gilbert Hocking, Bijectively related spaces. I.

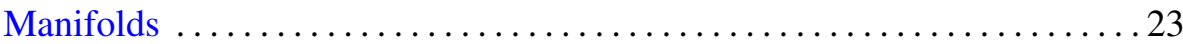

Joel Hass, Complete area minimizing minimal surfaces which are not totally

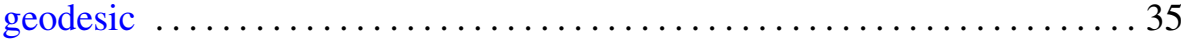

Aarno Hohti, On Ginsburg-Isbell derivatives and ranks of metric spaces .... 39

Richard Howard Hudson, Diophantine determinations of $3^{(p-1) / 8}$ and $5^{(p-1) / 4}$

A. F. Izé and A. Ventura, Asymptotic behavior of a perturbed neutral functional-differential equation related to the solution of the unperturbed

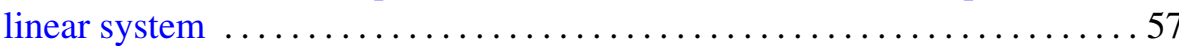

Palle E. T. Jorgensen, Spectral representations of unbounded nonlinear

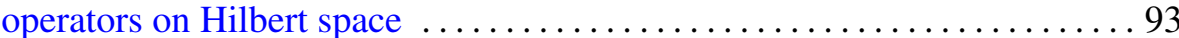

Darrell Conley Kent and Gary Douglas Richardson, Cauchy spaces with

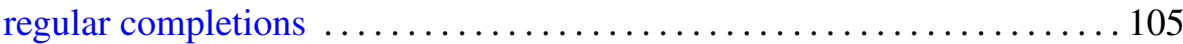

Mark Mahowald, An addendum to: "bo-resolutions" ................ 117

Stuart Wayne Margolis and Jean-Eric Pin, Minimal noncommutative varieties and power varieties

Carla Massaza and Alfio Ragusa, Some conditions on the homology groups of the Koszul complex

Vicente Miquel Molina, Some examples of Riemannian almost-product manifolds

Roderic Murufas, Inverse spectral problems for certain differential operators

Ulrich Oertel, Closed incompressible surfaces in complements of star links

Katsuro Sakai, A characterization of local equiconnectedness

William Victor Smith and Don Harrell Tucker, Weak integral convergence theorems and operator measures 